\title{
Influence of Nonlinear Intensity Attenuation in Bright-Field TEM Images on Tomographic Reconstructions of Micron-Scaled Materials
}

\author{
Jun Yamasaki ${ }^{1}$, Michihiro Mutoh ${ }^{2}$, Shigemasa Ohta ${ }^{3}$, Shuichi Yuasa ${ }^{3}$, Shigeo Arai $^{2}$, Katsuhiro Sasaki ${ }^{4}$ \\ and Nobuo Tanaka ${ }^{2}$ \\ 1. Research Center for Ultra-High Voltage Electron Microscopy, Osaka University, Ibaraki, Osaka, \\ Japan. \\ 2. EcoTopia Science Institute, Nagoya University, Nagoya, Japan. \\ 3. JEOL Ltd., Akishima, Tokyo, Japan. \\ 4. Department of Quantum Engineering, Nagoya University, Nagoya, Japan
}

Nowadays three-dimensional (3D) analyses of nanometer-sized and sub-micron-sized objects have been widely achieved by tomography in transmission electron microscopes (TEM). One of the next methodological targets should be quantitative 3D reconstructions in which not only the shape but also the internal density is correctly reproduced. This is, however, generally hindered by the nonlinearity between projection thickness and image intensity. In the case of mass-thickness contrast in bright-field TEM (BF-TEM) images, the ideal exponential attenuation with increasing thickness is disturbed by multiple scatterings. The nonlinearity in the tilt series should induce an inaccurate density distribution in the $3 \mathrm{D}$ reconstruction.

In the present study, the nonlinear attenuation effect has been analyzed using amorphous carbon microcoils (CMCs) [1] shown in Fig. 1(a). Their well-defined shapes and compositional homogeneity are quite useful to estimate the mass-thickness [2]. The intensity attenuation has been measured along the line in Fig. 1(b) taken by the high-voltage electron microscope (HVEM) [3]. The results measured at the acceleration voltages of $400,600,800$ and $1000 \mathrm{kV}$ are converted to the plots of the electron transmittance $T$ with increasing thickness in Fig. 2. At a glance, all the data seem to obey the linear attenuation regardless of the differences in the electron energy. However, the least square line for the data at $400 \mathrm{kV}$ has a considerable value of the negative intercept at zero thickness.

It is considered that such nonlinear attenuation should induce failures in conversion from intensity to thickness and thus inhibits correct 3D reconstructions. The influence of the nonlinearity on tomographic reconstructions has been examined using a specially-developed $360^{\circ}$-tilt sample holder for elimination of the missing-wedge effect [2]. Figure 3 shows the reconstruction results from the tilt series taken at $400 \mathrm{kV}$ and $1000 \mathrm{kV}$. Although the 3D shape of the CMC has been reconstructed well in both cases, the internal density is not uniform but has gradient from the center at $400 \mathrm{kV}$. Moreover, there is slight increase of the vacuum level at the inside region of the coil. The inaccurate density reconstruction should result from the nonlinearity pointed in Fig. 2. Judging from the plot for $600 \mathrm{kV}$ electrons in Fig. 2, the linearity is valid at least down to $\ln T$ of -0.4 , which corresponds to about $2 / 3$ of the electron transmittance. This information should be beneficial in actual tomography experiments of carbon-based materials because one can foresee quality of the reconstruction from the minimum transmittance in a single BF-TEM image prior to the tilt series acquisition.

\section{References:}

[1] S. Motojima et al., Appl. Phys. Lett. 56 (1990), p. 321. 
[2] J. Yamasaki et al., Microscopy, 63 (2014), p. 345.

[3] N. Tanaka et al., Microscopy 62 (2013), p. 205.
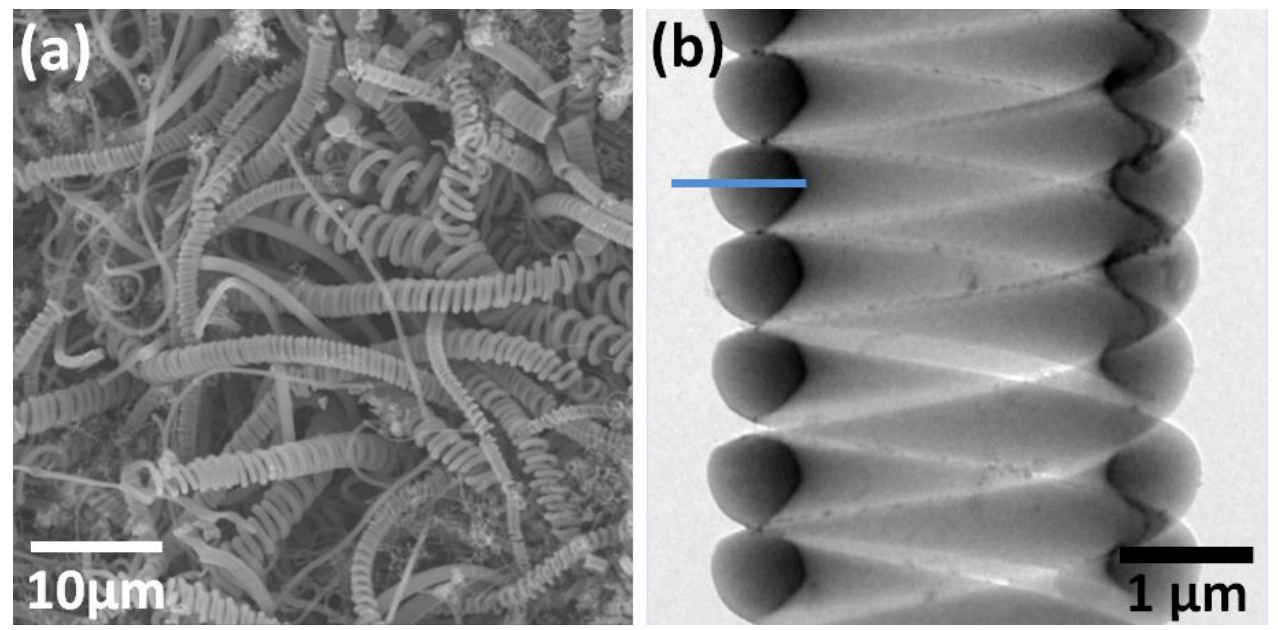

Figure 1. Carbon microcoils. (a) SEM image (taken by Microphase Co., Ltd.) and (b) BF-TEM image taken by HVEM.

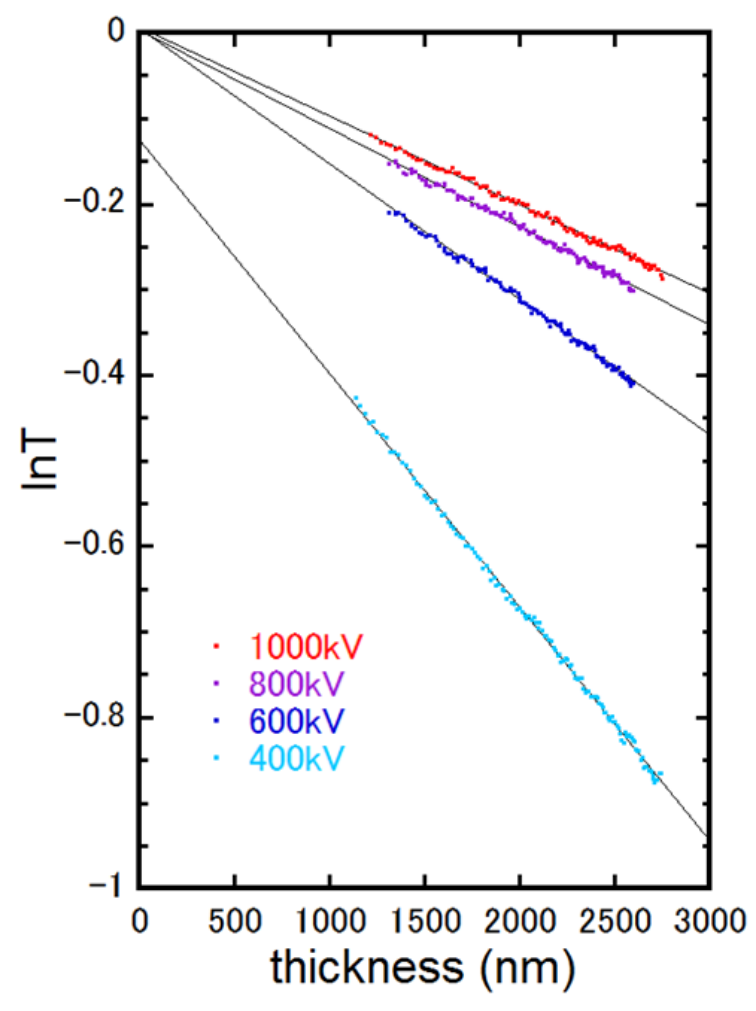

Figure 2. Attenuation of electron transmittance $T$ in BF-TEM images with increasing thickness.
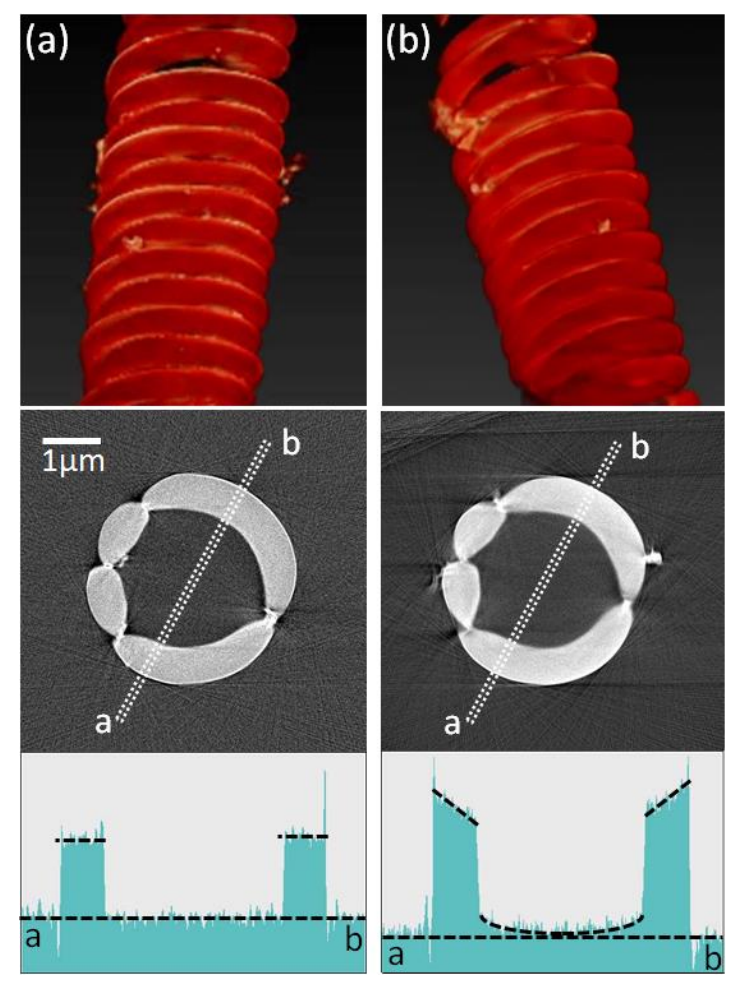

Figure 3. 3D reconstructions of the CMC from the tilt series of BF-TEM images taken at (a) $1000 \mathrm{kV}$ and (b) $400 \mathrm{kV}$. 